\title{
DISCRETE SUBSETS OF PERFECTLY NORMAL SPACES
}

\author{
R. M. STEPHENSON, JR.
}

\begin{abstract}
Following R. W. Heath and E. A. Michael [HM], we call a topological space perfect if each of its closed subsets is a $G_{\delta}$

In this note we (i) give a characterization of compact perfect $T_{1^{-}}$ spaces and (ii) obtain some results concerning the cardinality of discrete subsets of perfect spaces.
\end{abstract}

0. Preliminaries. We denote the cardinality of a set $X$ by $|X|$, and we denote $2 \boldsymbol{N}_{0}$ by $c$.

As used here, the concepts of compactness, normality, etc. do not require that the $T_{1}$-separation axiom be satisfied. By a countably compact space we will mean a space in which each countable open cover has a finite subcover. A space will be called a Lindelöf space if each open cover has a countable subcover.

Several ideas due to the referee are used below, namely, the proof of Theorem 2, and the observation that the conclusion of Theorem 3 holds in a more general situation than in just a countably compact, noncompact one.

1. A characterization of compact perfect $T_{1}$-spaces. In [GFW], S. L. Gulden, W. M. Fleischman, and J. H. Weston call a topological space $\aleph_{0}$-bounded if each of its countable subsets has compact closure.

Definition. We shall call a space $\boldsymbol{\aleph}_{0}$-discrete-bounded if each of its countable discrete subsets has compact closure.

For any topological space one can prove the following: compact $\Rightarrow \boldsymbol{\aleph}_{\mathbf{0}^{-}}$ bounded $\Rightarrow \boldsymbol{\aleph}_{0}$-discrete-bounded $\Rightarrow$ countably compact. In a metric space all these properties are equivalent. For the larger class of perfect spaces, however, it is apparently not known if countable compactness implies compactness. In Theorem 1 we obtain a result in that direction.

Theorem 1. Let $X$ be a $T_{1}$-space which is perfect and $\boldsymbol{\aleph}_{0}$-discretebounded. Then $X$ is compact.

Theorem 1 is an immediate consequence of Theorems 2 and 3.

Received by the editors August 6, 1971.

AMS 1970 subject classifications. Primary 54D15; Secondary 54D30.

Key words and phrases. Compact perfectly normal spaces, discrete subspaces, countably compact perfect spaces.

(C) American Mathematical Society 1972 
THEOREM 2. Let $X$ be a $T_{1}$-space which is perfect and countably compact. Then every discrete subset $Y$ of $X$ is countable.

Proof. Let $F$ be the set of all limit points of $Y$ in $X$. Then $F$ is closed in $X, Y \cap F=\varnothing$, and $Y$ is closed in $X \backslash F$. Since $X \backslash F$ is open in $X, X \backslash F$ is an $F_{\sigma}$ in $X$. Hence $Y$ is also an $F_{\sigma}$ in $X$. Because each closed discrete subspace of $X$ is finite, $Y$ must thus be countable.

THEOREM 3. Let $X$ be a space in which the closure of every countable discrete subset is a Lindelöf space, but suppose that $X$ itself is not Lindelöf. Then $X$ has an uncountable discrete subset.

Proof. Choose an open cover $\mathscr{U}$ of $X$ which has no countable subcover, and let $\mathscr{M}$ be the family of all mappings $f$ such that: (i) if $(x, V) \in f$ then $x \in X, V$ is a neighborhood of $x$ in $X$, and $V$ is contained in some member of $\mathscr{U}$; and (ii) if $(x, V),(y, W) \in f$ with $x \neq y$, then $x \notin W$ and $y \notin V$. Let $m$ be a maximal element of $(\mathscr{M}, \subset)$, and define $I=$ domain of $m$ and $\mathscr{D}=$ range of $m$.

Clearly $I$ is discrete, and so if it is uncountable, our proof is complete.

Let us suppose that $I$ is countable. Then $\bar{I}$ is Lindelöf, and hence $\bar{I} \subset \bigcup \mathscr{C}$ for some countable subcollection $\mathscr{C}$ of $\mathscr{U}$. By (i), $\bigcup \mathscr{D}$ is also covered by a countable subcollection of $\mathscr{U}$. Since no countable subcollection of $\mathscr{U}$ covers $X$, there must exist a point $x \in X$ such that $x \notin \bigcup \mathscr{C}$ and $x \notin \bigcup \mathscr{D}$. But if $U$ is any set in $\dddot{U}$ with $x \in U$ then $m \cup\{(x, U \cap(X \backslash I))\}$ is a member of $\mathscr{M}$ strictly greater than $m$.

2. The cardinality of discrete subsets of perfect spaces. If a perfectly normal space $X$ has a dense subset of cardinality $\mathfrak{M} \geqq \aleph_{0}$, then a simple counting argument shows that for every discrete subset $I$ of $X$, one must have $2^{|I|} \leqq 2^{\exists \jmath}$. (A variety of examples show that if $X$ is just perfect but not normal, then this inequality need not hold.)

In Theorem 2 we proved that if a perfect $T_{1}$-space satisfies a certain compactness condition, namely, countable compactness, then there is a sharper upper bound for the cardinalities of its discrete subsets. A related condition, one that is known to be implied by countable compactness, equivalent to it in normal spaces, and equivalent to pseudocompactness in completely regular spaces, is feeble compactness - the requirement that every locally finite system of open sets be finite. The example in [GJ, 5I] shows that in Theorem 2 countable compactness cannot be weakened to feeble compactness, even for a perfect, locally compact, zero-dimensional $T_{1}$-space; however, a proof similar to the proof of Theorem 2 shows that every open discrete subset of a perfect feebly compact space is countable.

It should be noted that, in the presence of the Continuum Hypothesis, Theorem 2 is known for the case in which the space $X$ is compact 
Hausdorff. For by a result of Alexandrov and Urysohn [AU2], every perfect compact Hausdorff space has no more than $c$ closed sets, and, obviously, no space with the latter property can have a discrete subset of cardinality $\geqq c$. We show next that the hypothesis of this theorem of Alexandrov and Urysohn (and also the hypotheses of the results established in [AU1, pp. 38-41]) can be weakened.

THEOREM 4. Let $X$ be a perfect $T_{1}$-space. (i) If $X$ is either Lindelöf or countably compact then $|X| \leqq c$. (ii) If $X$ is Lindelöf, and if each point $x \in X$ has a fundamental system of neighborhoods of cardinality $\leqq \mathfrak{c}$, then $X$ has no more than $\mathrm{c}$ closed sets.

Proof. (i) According to [HJ, Theorem 6], if $X$ is a space in which (a) each point is a $G_{\delta}$ and (b) each discrete subset is countable, then $|X| \leqq c$. Since here $X$ is $T_{1}$, perfect, and either countably compact or hereditarily Lindelöf, (a) and (b) are satisfied. (ii) Then $X$ has a base $\mathscr{B}$ with $|\mathscr{B}| \leqq c$, and every closed subset of $X$ can be obtained by taking a countable intersection of countable unions of members of $\mathscr{B}$.

The following space $X$ is a perfect, feebly compact, extremally disconnected Hausdorff space of cardinality $>c$.

EXAmple 5. Let $N \subset Y \subset \beta N$ with $Y$ pseudocompact and $|Y|>$ c. Let $X$ be the space whose points are those of $Y$, and which is topologized as follows: each point of $N$ is isolated; a neighborhood of a point $p \in X \backslash N$ is any superset of a set of the form $\{p\} \cup(N \cap F)$ where $F$ is a neighborhood of $p$ in $Y$.

We conclude by raising two questions. (i) Is there a regular perfect $T_{1}$-space that is countably compact but not compact? (ii) Is there a compact Hausdorff space which has no more than c closed sets but which is not perfect?

We observe that no subspace of a linearly ordered space can be used to obtain an affirmative answer to (i), for D. Lutzer [L] has proved that a perfect subspace of a linearly ordered space is hereditarily paracompact.

If $\mathrm{c}=\exp \left(\boldsymbol{\aleph}_{1}\right)$ then the ordered space consisting of all ordinals $\leqq \omega_{1}$ is a compact Hausforff space which is not perfect but in which there are no more than $c$ closed sets.

\section{REFERENCES}

[AU1] P. Alexandrov and P. Urysohn, Mémoire sur les espaces topologiques compacts, Verh. Kon. Akad. Wetensch. Amsterdam 14 (1929), no. 1,1-96.

[AU2] — "On compact topological spaces," in P. S. Urysohn, Works in topology and other fields of mathematics. Vol. 2, GITTL, Moscow, 1951. MR 14, 122.

[GJ] L. Gillman and M. Jerison, Rings of continuous functions, University Series in Higher Math., Van Nostrand, Princeton, N.J., 1960. MR 22 \#6994. 
[GFW] S. L. Gulden, W. M. Fleischman and J. H. Weston, Linearly ordered topological spaces, Proc. Amer. Math. Soc. 24 (1970), 197-203: MR 40 \#3511.

[HJ] A. Hajnal and I. Juhász, Discrete subspaces of topological spaces, Nederl. Akad. Wetensch. Proc. Ser. A 70 = Indag. Math. 29 (1967), 343-356: MR 37 \#4769.

[HM] R. W. Heath and E. A. Michael, A property of the Sorgenfrey line, Compositio Math. 23 (1971), 185-188.

[L] D. J. Lutzer, On generalized ordered spaces, Proc. Washington State Univ. Conf. on General Topology (Pullman, Wash., 1970), Pi Mu Epsilon, Dept. of Math., Washington State Univ., Pullman, Wash., 1970, pp. 102-110. MR 42 \#1075.

Department of Mathematics, University of North Carolina, Chapel Hill, North CAROLINA 27514 